\title{
Efficacy of vitrectomy and epiretinal membrane peeling in eyes with dry age-related macular degeneration
}

\author{
John O Mason III', \\ Shyam A Patel' \\ 'Department of Ophthalmology, \\ University of Alabama School of \\ Medicine, Birmingham, AL, USA; \\ ${ }^{2}$ Retina Consultants of Alabama, \\ Callahan Eye Foundation Hospital, \\ Birmingham, AL, USA
}

This article was published in the following Dove Press journal:

Clinical Ophthalmology

23 October 2015

Number of times this article has been viewed

Objective: To study the efficacy of epiretinal membrane (ERM) peeling in eyes with dry agerelated macular degeneration (AMD).

Methods: We retrospectively analyzed patient charts on 17 eyes (16 patients) that underwent ERM peeling with a concurrent diagnosis of dry AMD.

Results: Eyes with concurrent dry AMD and with a good preoperative best-corrected visual acuity (BCVA) (better than or equal to 20/50) had a statistically significant mean BCVA improvement at 6 months after ERM peeling. There was a statistical increase in mean BCVA from 20/95 to 20/56 in dry AMD eyes, and no eyes showed worsening in BCVA at 6 months or at most recent follow-up. Five/seventeen (29.4\%) eyes had cataract formation or progression. There were no other complications, reoperations, or reoccurrences.

Conclusion: ERM peeling in eyes with dry AMD may show significant improvement, especially in eyes with good preoperative BCVA. The procedure is relatively safe with low complications and reoccurrences.

Keywords: macular pucker, epiretinal membrane peeling, epimacular membrane, macular degeneration

\section{Introduction}

Epiretinal membrane (ERM), also known as macular pucker or epimacular membrane, consists of an avascular layer of cells that form and overly the macula. This membrane may create tension on the retina leading to a decrease in visual acuity, distortion in central vision, blurriness, and binocular diplopia.

Vitrectomy with ERM peeling has shown to be beneficial, even in eyes with good preoperative visual acuity. ${ }^{1}$ Dawson et al concluded that $70 \%$ of their 237 patients showed improvement in visual acuity, and only $15.2 \%$ showed a decrease in visual acuity after vitrectomy and ERM peeling. They also showed that postoperative visual acuity was directly related to preoperative visual acuity. ${ }^{2}$ Other prognostic indicators for better outcomes are undergoing combined cataract surgery with vitrectomy for phakic eyes, early timing of ERM treatment after symptom onset, and pseudophakic lens status at 3 months after surgery. ${ }^{3-5}$ In addition, contrast sensitivity improves in eyes following vitrectomy for ERM, and central macular thickness (CMT) has been shown to decrease (improve) following ERM peeling by approximately an average of $120 \mu \mathrm{m}$ following surgery. ${ }^{6,7}$

After a Medline search, we could find very limited data evaluating the effects of vitrectomy and ERM peeling in eyes with dry age-related macular degeneration (AMD). Since both pathologies affect the macula, many surgeons may be reluctant in performing 
vitrectomy as they may question the benefits of removing an ERM in a patient with underlying AMD. Roller et al conducted a study assessing the effects of vitrectomy on eyes with AMD, and a subgroup of 13 eyes of the 22 total eyes studied underwent vitrectomy for ERM specifically. Of these 13 eyes with ERM and AMD, five (38.5\%) eyes had progression of AMD, five (38.5\%) had improvement in visual acuity, and six $(46.2 \%)$ eyes showed a decrease in postoperative visual acuity. ${ }^{8}$ These are considerably worse results compared to the previously mentioned ERM peeling eyes without AMD.

In this study, we retrospectively sought to evaluate the efficacy of ERM peeling in eyes with category 1 or category 2 dry AMD, and we compare these results to previous studies on ERM peeling.

\section{Methods}

We retrospectively evaluated 17 eyes (16 patients) with a dry AMD diagnosis (category 1 or category 2 dry AMD) that were treated with non-combined (without concurrent cataract surgery) 25-gauge pars plana vitrectomy with ERM peeling from 2010 to 2012 at the Retina Consultants of Alabama and at the University of Alabama at Birmingham Callahan Eye Hospital. All procedures were performed by one of the four practicing retina specialists. All procedures included the peeling of the internal limiting membrane (ILM) in conjunction with ERM peeling. After removal of the ERM, indocyanine green dye $0.05 \mathrm{mg} / \mathrm{mL}$ was placed on the macula for 15 seconds to allow for minimally perceptible staining of the ILM prior to removal. Institutional Review Board at University of Alabama at Birmingham approved this study, and this study is HIPAA compliant. Patient consent was not necessary as HIPAA approval was provided by the University of Alabama at Birmingham.

Patient charts were analyzed for multiple variables including sex, age, left versus right eye, pre-existing ocular conditions, presence of retinal tear or posterior vitreous detachment before surgery, lens status (phakic or pseudophakic), preoperative and postoperative (within 6 months) CMT via optical coherence tomography (OCT), preoperative and postoperative best-corrected visual acuities (BCVAs), and intraocular pressures. Additionally, intraoperative complications, postoperative complications, reoccurrences, and reoperations were also recorded.

Primary outcome measures include postoperative BCVA and the BCVA change compared to baseline preoperative BCVA. Secondary outcomes include the number of eyes with a BCVA better or equal to 20/50, the mean difference in CMT, complication rates, reoccurrence rates, and reoperation rates.
Statistical analysis (means, differences, and $P$-values) of the data was conducted by using Microsoft Excel 2011. Snellen chart visual acuity values were converted in logarithm of the minimum angle of resolution ( $\log$ MAR) values. BCVA improvement and worsening were defined as two or more Snellen lines better/worse than the baseline preoperative BCVA. $P$-values for continuous data (CMT and logMAR values) were calculated by using the paired Student's $t$-test method. $P$-values for categorical data were calculated by using the chi-squared method. $P$-values less than 0.05 are considered to be statistically significant.

\section{Results}

Seventeen eyes (16 patients) with dry AMD had 25-gauge pars plana vitrectomy with ERM peeling. All patients had a recorded BCVA at 1 month, 3 months and/or 6 months postoperatively, and the mean follow-up period was 5.1 months (range: $3-6$ months). The mean age was 74.9 years (range: 65-83 years) (Table 1). Seven eyes were males, and eleven eyes were right eyes. Eight eyes had a posterior vitreous detachment noted on exam prior to surgery, and eleven eyes were pseudophakic preoperatively, while six were phakic. There were no retinal tears in the posterior pole or periphery noted prior to operation.

Table I Baseline characteristics in eyes with dry age-related macular degeneration undergoing vitrectomy with epiretinal membrane peeling

\begin{tabular}{ll}
\hline Characteristic & Eyes \\
\hline Number of eyes & 17 \\
Age (years) & \\
Mean & 74.9 \\
Standard deviation & 5.9 \\
Range & $65-83$ \\
Sex & \\
Male & $7(41.2 \%)$ \\
Female & $10(58.8 \%)$ \\
Eye & \\
Right & $11(64.7 \%)$ \\
Left & $6(35.3 \%)$ \\
Lens status & \\
Phakic & $6(35.3 \%)$ \\
Pseudophakic & $11(64.7 \%)$ \\
Pre-existing ocular condition & \\
Retinal tear & $0(0 \%)$ \\
Posterior vitreous detachment & $8(47.1 \%)$ \\
Cataract & $2(33.3 \%)^{*}$ \\
Glaucoma & $0(0 \%)$ \\
Diabetic retinopathy & $0(0 \%)$ \\
Hypertensive retinopathy & $2(11.8 \%)$ \\
\hline
\end{tabular}

Note: *Cataract percentage was calculated by using the total number of eyes having phakic lens status. 
Table 2 Baseline and postoperative best-corrected visual acuities in eyes undergoing epiretinal membrane peeling

\begin{tabular}{|c|c|c|c|c|c|}
\hline & Preoperative & I month & 3 months & 6 months & Most recent follow-up* \\
\hline Total eyes & 17 & 16 & 15 & 12 & 17 \\
\hline Eyes with BCVA improvement & - & 7 & 8 & 6 & 10 \\
\hline Eyes with no BCVA change & - & 9 & 6 & 6 & 7 \\
\hline Eyes with BCVA worsening & - & 0 & I & 0 & 0 \\
\hline Mean logMAR & 0.68 & 0.52 & 0.44 & 0.49 & 0.45 \\
\hline Standard deviation & 0.45 & 0.34 & 0.30 & 0.40 & 0.001 \\
\hline Mean Snellen score & $20 / 95$ & $20 / 70$ & $20 / 55$ & $20 / 61$ & $20 / 56$ \\
\hline$P$-value & - & 0.32 & 0.15 & 0.26 & 0.001 \\
\hline Mean logMAR in eyes with a preoperative & $0.36(n=7)$ & $0.28(n=7)$ & $0.24(n=7)$ & $0.21(n=5)$ & $0.21(n=7)$ \\
\hline \multicolumn{6}{|l|}{ visual acuity $\geq$ (better or equal to) $20 / 50$} \\
\hline Mean Snellen score & $20 / 46$ & $20 / 42$ & $20 / 38$ & $20 / 32$ & $20 / 32$ \\
\hline$P$-value & - & 0.14 & 0.12 & 0.046 & $<0.001$ \\
\hline Mean logMAR in eyes with a preoperative & $0.90(n=10)$ & $0.70(n=9)$ & $0.61(n=8)$ & $0.68(n=7)$ & $0.63(n=10)$ \\
\hline \multicolumn{6}{|l|}{ visual acuity $<$ (worse than) $20 / 50$} \\
\hline Mean Snellen score & $20 / 159$ & $20 / 100$ & $20 / 81$ & $20 / 96$ & $20 / 85$ \\
\hline$P$-value & - & 0.11 & 0.35 & 0.41 & 0.02 \\
\hline Pseudophakic lens status & $0.58(n=I I)$ & $0.45(n=11)$ & $0.43(n=11)$ & $0.38(n=7)$ & $0.38(n=I 1)$ \\
\hline Mean Snellen score & $20 / 76$ & $20 / 56$ & $20 / 54$ & $20 / 48$ & $20 / 48$ \\
\hline$P$-value & - & 0.01 & 0.04 & 0.26 & 0.005 \\
\hline Phakic lens status & $0.85(n=6)$ & $0.45(n=5)$ & $0.45(n=4)$ & $0.64(n=5)$ & $0.25(n=6)$ \\
\hline Mean Snellen score & $20 / 142$ & $20 / 56$ & $20 / 56$ & $20 / 87$ & $20 / 36$ \\
\hline$P$-value & - & 0.92 & 0.18 & 0.98 & 0.02 \\
\hline
\end{tabular}

Notes: *Most recent follow-up includes the last time the patient was seen during the 6-month time frame postoperatively. All $P$-values are calculated in comparison to preoperative status.

Abbreviations: BCVA, best-corrected visual acuity; logMAR, logarithm of the minimum angle of resolution.

At most recent follow-up, 10/17 (59\%) eyes showed improvement in BCVA and 7/17 (41\%) showed no change (Table 2). No eyes showed worsening in BCVA at the most recent follow-up. BCVA improved in six eyes at 6 months and worsened in no eyes at 6 months. While six eyes showed no change in BCVA at 6 months, the difference is attributable to cataract formation or progression. The mean BCVA change was only statistically significant at 6 months in eyes with a preoperative BCVA better than or equal to 20/50 $(\log M A R=0.4)$. When using most recent follow-up, BCVA improvement was statistically significant, regardless of lens status or preoperative BCVA. Table 3 shows that the number of eyes with a BCVA better than or equal to 20/50 increased postoperatively during the study, and this finding was statistically significant. The average preoperative and postoperative intraocular pressure (at $1 \mathrm{month}$ ) was $14.5 \mathrm{mmHg}$ and $15.2 \mathrm{mmHg}$, respectively.

Preoperative and postoperative OCT data were available for nine eyes (Table 4). All nine eyes had a decrease

Table 3 Number of eyes with a visual acuity equal to or better than $20 / 50$

\begin{tabular}{llll}
\hline & Preoperative & Most recent follow-up & P-value \\
\hline Number of eyes & 7 & 12 & 0.039 \\
\hline
\end{tabular}

Note: Most recent follow-up includes the last time the patient was seen during the 6-month time frame postoperatively. in macular thickness, with a mean CMT decrease of $93 \mu \mathrm{m}$, which was statistically significant. The change in the mean CMT in eyes with a BCVA improvement was statistically significant. However, it was not significant in eyes with no change in BCVA. Even though the $R$-squared value for eyes with BCVA improvement was 0.76 , the $P$-value for the correlation was 0.85 . Six of 17 eyes had preoperative intraretinal macular edema, which resolved clinically and by OCT in 5/6 (83\%) eyes after surgery (Figure 1).

Nuclear sclerotic cataract formation and progression were found in 5/6 (83\%) phakic eyes at the end of follow-up (Table 5). Only $2 / 6$ phakic eyes underwent cataract extraction. There were no other complications, reoccurrences of macular pucker, or reoperations. No eyes developed a choroidal neovascular membrane.

\section{Discussion}

When comparing our results to Dawson et al's study of 237 patients undergoing ERM peeling without underlying dry AMD, the percentage of eyes with visual acuity improvement was quite similar. Using the most recent postoperative visual acuity, 10/17 (59\%) eyes in our study showed BCVA improvement while no eyes showed a decrease in BCVA. This is similar to the 70\% BCVA improvement in Dawson et al's study. Our results are dramatically different from 
Table 4 Comparison of preoperative and postoperative central macular thickness (OCT) in eyes undergoing epiretinal membrane peeling

\begin{tabular}{|c|c|c|c|}
\hline & Preoperative CMT & Postoperative CMT & Difference in CMT \\
\hline All eyes $(n=9)$ & 413 & 320 & 93 \\
\hline$P$-value & - & 0.01 & - \\
\hline Eyes with an improvement in visual acuity $(n=6)$ & 435 & 339 & 96 \\
\hline$P$-value & - & 0.01 & - \\
\hline Eyes with no change in visual acuity $(n=3)$ & 371 & 281 & 90 \\
\hline$P$-value & - & 0.19 & - \\
\hline
\end{tabular}

Notes: Visual improvement was based on most recent follow-up data. The units for central macular thickness numbers in the table are $\mu \mathrm{m}$.

Abbreviations: CMT, central macular thickness; OCT, optical coherence tomography.

Roller et al's study on eyes undergoing membrane peeling with AMD. Our eyes showed drastically more improvement as Roller's study showed improvement in only $38 \%$ of eyes. Our mean difference in BCVA was a $0.23 \log$ MAR improvement at the most recent follow-up, compared to only a $0.10 \log$ MAR improvement in Roller's study. ${ }^{8}$

In addition, our study agrees with Lehpamer and Carvounis' study on eyes with good preoperative visual acuity undergoing ERM peeling. Their study showed that eyes with a preoperative BCVA better than or equal to 20/50 had $0.093 \log$ MAR improvement at 6 months. ${ }^{1}$ Our eyes with good preoperative BCVA (BCVA better than or equal to 20/50) had a statistically significant improvement of 0.15 $\log$ MAR at 6 months. Eyes with worse preoperative visual acuity showed no significant change in BCVA at 6 months. This may be because the dry AMD in eyes with good BCVA may be not as advanced as in eyes with poorer BCVA, thereby limiting the visual improvement.

The average CMT decreased by $93 \mu \mathrm{m}$, which equates to a $22.8 \%$ change. This is significantly more than the $8.2 \%$ decrease at 6 months reported in Lehpamer and Carvounis' study. ${ }^{1}$ This average decrease was statistically significant in the specific population with a BCVA improvement, suggesting that BCVA improvement may be related to a decrease in CMT, even though the correlation between these two factors was not statistically significant in our sample of six eyes. It should be noted that the parameters of the outer retina were not addressed in this study. In addition, macular edema resolved in five eyes out of six eyes with preoperative intraretinal macular edema. Using the most recent visual acuity, the BCVA improvement in this population was $0.23 \log \mathrm{MAR}$, while all other eyes had a visual acuity improvement of $0.22 \log$ MAR. The one patient with persistent intraretinal macular edema had no change in visual acuity.

Lehpamer and Carvounis' study of eyes with preoperative visual acuities of 20/50 or better revealed that six of $13(46.1 \%)$ phakic eyes had progression or formation of cataracts postoperatively. ${ }^{1}$ We showed a higher rate as $83.3 \%$ (five out of six) of the phakic eyes had progression or formation of cataract. Of these five eyes, two underwent phacoemulsification within the follow-up period. Post-vitrectomy cataract progression is thought to be linked to an increase in oxygen tension exposed to the lens. This eventually leads to an increase in oxidation, either directly or through free radicals, which is proposed to be the cause of cataract progression. ${ }^{9}$

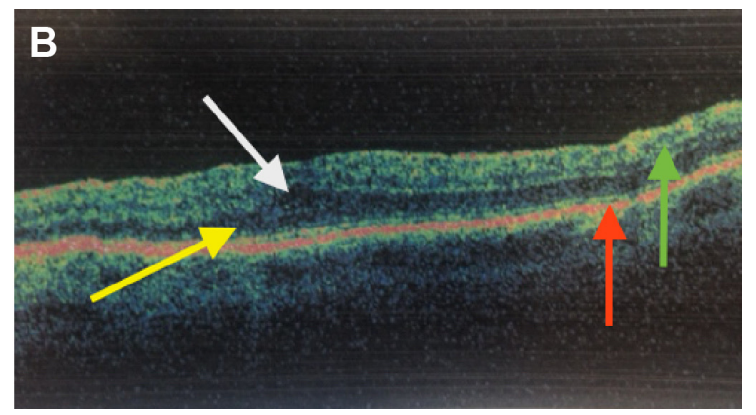

Figure I Improvement in central macular thickness on optical coherence tomography after undergoing epiretinal membrane peeling.

Notes: (A) A preoperative OCT showing an ERM with significant macular edema (yellow arrow), an intraretinal cyst (white arrow), and a CMT of $399 \mu$ m. (B) A postoperative OCT of the same eye showing the removal of the ERM and resolution of macular edema with a CMT of $32 \mathrm{I} \mu \mathrm{m}$. (OCT figures are scaled to $5.0 \mathrm{~mm}$ in length. Red arrow represents retinal pigment epithelium, and green arrow represents nerve fiber layer).

Abbreviations: OCT, optical coherence tomography; ERM, epiretinal membrane; CMT, central macular thickness. 
Table 5 Complications, reoccurrences, and reoperations in eyes undergoing epiretinal membrane peeling

Eyes $(n=17)$

\begin{tabular}{ll}
\hline Complications & \\
Retinal detachments & 0 \\
Formation or progression of cataract & 5 \\
Hemorrhage & 0 \\
Other & 0 \\
Epiretinal membrane reoccurrence & 0 \\
Reoperation & 0 \\
\hline
\end{tabular}

A recent study also reveals that increased cytokines in eyes with prior vitrectomy may contribute to cataract formation. ${ }^{10}$ There were no infections, retinal tears, detachments, recurrences, and reoperations in the 33 eyes studied by Lehpamer and Carvounis. ${ }^{1}$ Similarly, our data showed no complications, reoperations, or recurrences.

Limitations of this study include its retrospective nature, lack of control group, the possible different pathogenesis of idiopathic ERM compared to ERM in patients with AMD, and its short follow-up period (6 months). Although very few patients did not have all the information at specific follow-up dates postoperatively, our study numbers (even though more than Roller's study) are not considered to be a large sample size. In addition, there may have been some variations from surgeon to surgeon when performing the surgery. Subjective components of certain characteristics, such as the progression of cataracts add variation to the data as well. However, our visual results might have been even better if all patients who had progression or formation of cataract underwent phacoemulsification.

Eyes with category 1 and 2 dry AMD may benefit from ERM peeling, and a reduction of CMT is statistically significant in eyes with BCVA improvement. The BCVA improvement after ERM peeling may be greater than what was previously thought in eyes with dry AMD. In addition, the procedure has a low complication, reoperation, and recurrence rate. We would recommend further studies, preferably prospective, that analyze ERM peeling in this population of patients with dry AMD.

\section{Disclosure}

The authors have no conflicts of interest to disclose.

\section{References}

1. Lehpamer BP, Carvounis PE. Pars Plana Vitrectomy for symptomatic epiretinal membrane in eyes with $20 / 50$ or better preoperative visual acuity. Retina. 2015;35(9):1822-1827.

2. Dawson SR, Shunmugam M, Williamson TH. Visual acuity outcomes following surgery for idiopathic epiretinal membrane: an analysis of data from 2001 to 2011. Eye (Lond). 2014;28(2):219-224.

3. Song SJ, Kuriyan AE, Smiddy WE. Results and prognostic factors for visual improvement after pars plana vitrectomy for idiopathic epiretinal membrane. Retina. 2015;35(5):866-872.

4. Rahman R, Stephenson J. Early surgery for epiretinal membrane preserves more vision for patients. Eye (Lond). 2014;28(4):410-414.

5. Lee PY, Cheng KC, Wu WC. Anatomic and functional outcome after surgical removal of idiopathic macular epiretinal membrane. Kaohsiung J Med Sci. 2011;27(7):268-275.

6. Sugiura Y, Okamoto F, Okamoto Y, Hiraoka T, Oshika T. Contrast sensitivity and foveal microstructure following vitrectomy for epiretinal membrane. Invest Ophthalmol Vis Sci. 2014;55(11):7594-7600.

7. Massin P, Allouch C, Haouchine B, et al. Optical coherence tomography of idiopathic macular epiretinal membranes before and after surgery. Am J Ophthalmol. 2000;130(6):732-739.

8. Roller AB, Mahajan VB, Boldt HC, Abramoff MD, Russell SR, Folk JC. Effects of vitrectomy on age-related macular degeneration. Ophthalmology. 2010;117(7):1381-1386.

9. Holekamp NM, Shui YB, Beebe DC. Vitrectomy surgery increase oxygen exposure to the lens: a possible mechanism for nuclear cataract formation. Am J Ophthalmol. 2005;139(2):302-310.

10. Gu R, Zhou M, Jiang C, Yu J, Xu G. Elevated concentration of cytokines in aqueous humor in post-vitrectomy eyes. Clin Experiment Ophthalmol. Epub 2015 Aug 28.
Clinical Ophthalmology

\section{Publish your work in this journal}

Clinical Ophthalmology is an international, peer-reviewed journal covering all subspecialties within ophthalmology. Key topics include: Optometry; Visual science; Pharmacology and drug therapy in eye diseases; Basic Sciences; Primary and Secondary eye care; Patient Safety and Quality of Care Improvements. This journal is indexed on

Submit your manuscript here: http://www.dovepress.com/clinical-ophthalmology-journal

\section{Dovepress}

PubMed Central and CAS, and is the official journal of The Society of Clinical Ophthalmology (SCO). The manuscript management system is completely online and includes a very quick and fair peer-review system, which is all easy to use. Visit http://www.dovepress.com/ testimonials.php to read real quotes from published authors. 